Research Article

\title{
Strong Convergence Algorithms of the Split Common Fixed Point Problem for Total Quasi-Asymptotically Pseudocontractive Operators
}

\author{
Peiyuan Wang and Hy Zhou \\ Department of Mathematics, Ordnance Engineering College, Shijiazhuang, Hebei 050003, China \\ Correspondence should be addressed to Peiyuan Wang; wangpy629@163.com \\ Received 19 January 2014; Revised 2 March 2014; Accepted 16 March 2014; Published 3 April 2014 \\ Academic Editor: Jaan Janno
}

Copyright (C) 2014 P. Wang and H. Zhou. This is an open access article distributed under the Creative Commons Attribution License, which permits unrestricted use, distribution, and reproduction in any medium, provided the original work is properly cited.

We present a new algorithm for solving the two-set split common fixed point problem with total quasi-asymptotically pseudocontractive operators and consider the case of quasi-pseudocontractive operators. Under some appropriate conditions, we prove that the proposed algorithms have strong convergence. The results presented in this paper improve and extend the previous algorithms and results of Censor and Segal (2009), Moudafi (2011 and 2010), Mohammed (2013), Yang et al. (2011), Chang et al. (2012), and others.

\section{Introduction}

Let $C$ and $Q$ be nonempty closed convex subsets of real Hilbert spaces $H_{1}$ and $H_{2}$, respectively. Let $A: H_{1} \rightarrow H_{2}$ be a bounded linear operator. To allow for constraints both in the domain and range of $A$, Censor and Elfving [1] originally formulated the split feasibility problem (SFP), which is to find a member of set

$$
\Omega=\{x \in C: A x \in Q\} \neq \emptyset .
$$

A recent generalization, due to Censor and Segal in [2], is called the split common fixed point problem (SCFPP), which is to find a point $x^{*}$ satisfying

$$
x^{*} \in C:=\bigcap_{i=1}^{t} \operatorname{Fix}\left(U_{i}\right), \quad A x^{*} \in Q:=\bigcap_{j=1}^{r} \operatorname{Fix}\left(T_{j}\right),
$$

where $U_{i}: H_{1} \rightarrow H_{1}(i=1,2, \ldots t)$ and $T_{j}: H_{2} \rightarrow H_{2}(j=$ $1,2, \ldots, r)$ are some nonlinear operators and $A: H_{1} \rightarrow H_{2}$ is also a bounded linear operator. Denote the solution set of SCFPP by

$$
\Gamma=\left\{x^{*} \in C \mid A x^{*} \in Q\right\} .
$$

In particular, if $t=r=1$, problem (2) is reduced to the two-set SCFPP, where $C:=\operatorname{Fix}(U)$ and $Q:=\operatorname{Fix}(T)$, and the SFP can be retrieved by picking as operators $U$ and $T$ orthogonal projections.

Censor and Segal [2] invented the following CQalgorithm with directed operators to solve the two-set SCFPP:

$$
\forall x_{0} \in H_{1}, \quad x_{n+1}=U\left(x_{n}-\gamma A^{*}(I-T) A x_{n}\right), \quad n \geq 0,
$$

where $x_{0} \in H$ and $\gamma \in(0,2 / L) ; L$ is the largest eigenvalue of the matrix $A^{*} A$.

Inspired by the work of Censor and Segal, for $\alpha_{n} \in(0,1)$, Moudafi presented the following iteration with the demicontractive mappings and quasi-nonexpansive operators in papers [3] and [4], respectively:

$$
\begin{gathered}
u_{n}=x_{n}-\gamma A^{*}(I-T) A x_{n}, \\
x_{n+1}=\left(1-\alpha_{n}\right) u_{n}+\alpha_{n} U\left(u_{n}\right), \quad \forall x_{0} \in H_{1}, n \geq 0 .
\end{gathered}
$$

Moudafi's results are weak convergence. In [5, 6], Mohammed utilized the strongly quasi-nonexpansive operators and quasi-nonexpansive operators to solve recursion (5) and obtain weak and strong convergence, respectively. Strong 
convergence of (5) with pseudo-demicontractive and firmly pseudo-demicontractive mappings can be found in $[7,8]$. Furthermore, for several different strong convergence recursions with nonexpansive operators for solving the SCFPP see $[9,10]$. For the purpose of generalization, papers [11-13] discussed the total asymptotically strictly pseudocontractive mappings and asymptotically strict pseudocontractive mappings for solving (2) and multiple-set fixed point problem (MSSFP) by the following iteration:

$$
\begin{gathered}
u_{n}=x_{n}-\gamma A^{*}\left(I-T^{n}\right) A x_{n}, \\
x_{n+1}=\left(1-\alpha_{n}\right) u_{n}+\alpha_{n} U^{n}\left(u_{n}\right), \quad \forall x_{1} \in H_{1}, \quad n \geq 1,
\end{gathered}
$$

which is of weak convergence; when $U$ is semicompact, strong convergence of (6) can be deduced. Obviously, (5) is the particular case of (6). On the other hand, papers $[14,15]$ presented cyclic algorithms of the SCFPP for directed operators and demicontractive mappings, and the results converge weakly.

However, we found that the strong convergence of (6) needs the condition of $U$ to be semicompact. In order to obtain strong algorithm for the two-set SCFPP without more constraints on $U$ or $T$ and continue to generalize the operators, in this paper, we propose a different iteration, which can ensure the strong convergence with more general case when the operators are total quasi-asymptotically pseudocontractive, demiclosed at the origin. We can choose an initial data $x_{1} \in H_{1}$ arbitrarily and define the sequence $\left\{x_{n}\right\}$ by the recursion:

$$
\begin{gathered}
u_{n}=x_{n}-\gamma A\left(I-T^{n}\right) A x_{n}, \\
y_{n}=(1-\beta) u_{n}+\beta U^{n}\left(u_{n}\right), \\
x_{n+1}=\alpha_{n} \psi\left(y_{n}\right)+\left(1-\alpha_{n}\right) y_{n}, \quad n \geq 1,
\end{gathered}
$$

where $\psi: H_{1} \rightarrow H_{1}$ is a $\delta$-contraction with $\delta \in(0,1)$, $T$ and $U$ are total quasi-asymptotically pseudocontractive mappings, and $\left\{\alpha_{n}\right\},\left\{\beta_{n}\right\}$, and $\left\{\gamma_{n}\right\}$ are three real sequences satisfying appropriate conditions. Under some mild conditions, we prove that the sequence $\left\{x_{n}\right\}$ generated by (7) converges strongly to the solution of the two-set SCFPP.

\section{Preliminaries}

In order to reach the main results, we first recall the following facts.

Let $C$ be a nonempty closed and convex subset of a real Hilbert space $H$ with the inner product $\langle\cdot, \cdot\rangle$ and norm $\|\cdot\|$. Denote by $\operatorname{Fix}(T)$ the set of fixed points of a mapping $T$; that is, $\operatorname{Fix}(T)=\{x \in C: T x=x\}$.

Definition 1 (see $[2,3,16,17]$ ). (i) Recalled that $T: C \rightarrow C$ is said to be a directed or firmly quasi-nonexpansive operator; if $p \in \operatorname{Fix}(T)$, then

$$
\|T x-p\|^{2} \leq\|x-p\|^{2}-\|x-T x\|^{2}, \quad \forall x \in C .
$$

(ii) Let $D$ be a closed convex nonempty set of $C$; $T: C \rightarrow$ $C$ is nonexpansive; we say that $T$ is attracting with respect to $D$, if, for every $x \in C \backslash D, p \in D$,

$$
\|T x-p\|<\|x-p\| \text {. }
$$

(iii) A mapping $T: C \rightarrow C$ is said to be paracontracting or quasi-nonexpansive; if $p \in \operatorname{Fix}(T)$, then

$$
\|T x-p\| \leq\|x-p\| \text {. }
$$

(iv) A mapping $T: C \rightarrow C$ is said to be demicontractive or strictly quasi-pseudocontractive; for $p \in \operatorname{Fix}(T)$, there exists a constant $\beta \in[0,1)$ such that

$$
\|T x-p\|^{2} \leq\|x-p\|^{2}+\beta\|x-T x\|^{2}, \quad \forall x \in C .
$$

Definition 2 (see $[11,18]$ ). (i) Let $T: C \rightarrow C$ be a total quasi-asymptotically strictly pseudocontractive if $F(T) \neq \emptyset$, and there exist a constant $\beta \in[0,1]$, sequences $\left\{\mu_{n}\right\} \subset[0, \infty)$, and $\left\{\xi_{n}\right\} \subset[0, \infty)$ with $\mu_{n} \rightarrow 0$ and $\xi_{n} \rightarrow 0$ as $n \rightarrow \infty$ such that

$$
\begin{array}{r}
\left\|T^{n} x-p\right\|^{2} \leq\|x-p\|^{2}+\beta\left\|x-T^{n} x\right\|^{2}+\mu_{n} \phi(\|x-p\|)+\xi_{n}, \\
\forall n \geq 1, x \in C, p \in \operatorname{Fix}(T),
\end{array}
$$

where $\phi:[0, \infty) \rightarrow[0, \infty)$ is a continuous and strictly increasing function with $\phi(0)=0$.

(ii) A mapping $T: C \rightarrow C$ is said to be total quasiasymptotically pseudocontractive if $F(T) \neq \emptyset$, and there exist sequences $\left\{\mu_{n}\right\} \subset[0, \infty)$ and $\left\{\xi_{n}\right\} \subset[0, \infty)$ with $\mu_{n} \rightarrow 0$ and $\xi_{n} \rightarrow 0$ as $n \rightarrow \infty$ such that

$$
\begin{array}{r}
\left\|T^{n} x-p\right\|^{2} \leq\|x-p\|^{2}+\left\|x-T^{n} x\right\|^{2}+\mu_{n} \phi(\|x-p\|)+\xi_{n}, \\
\forall n \geq 1, x \in C, p \in \operatorname{Fix}(T),
\end{array}
$$

where $\phi:[0, \infty) \rightarrow[0, \infty)$ is a continuous and strictly increasing function with $\phi(0)=0$.

(iii) A mapping $T: C \rightarrow C$ is said to be quasipseudocontractive if $\operatorname{Fix}(T) \neq \emptyset$, such that

$$
\|T x-p\|^{2} \leq\|x-p\|^{2}+\|x-T x\|^{2}, x \in C, p \in \operatorname{Fix}(T) .
$$

(iv) A mapping $T: C \rightarrow C$ is said to be uniformly $k$ Lipschitzian if there is a constant $k>0$, such that

$$
\left\|T^{n} x-T^{n} y\right\| \leq k\|x-y\|, \quad \forall n \geq 1, \forall x, y \in C .
$$

Remark 3. Note that the classes of directed operators and attracting operators belong to the class of paracontracting operators. The class of paracontracting operators belongs to the class of demicontractive operators, while the class of quasi-pseudocontractive operators includes the class of demicontractive operators. Further, the class of total quasiasymptotically pseudocontractive operators, with quasipseudocontractive operators as a special case, includes the class of total quasi-asymptotically strictly pseudocontractive operators. 
Remark 4. Let $T: C \rightarrow C$ be a total quasi-asymptotically pseudocontractive, if $F(T) \neq \emptyset$, for each $x \in C$ and $q \in$ $\operatorname{Fix}(T)$; from (13) we can easily obtain the following equivalent inequalities:

$$
\begin{gathered}
\left\langle x-T^{n} x, x-p\right\rangle \geq-\frac{\mu_{n}}{2} \phi(\|x-p\|)-\frac{\xi_{n}}{2} ; \\
\left\langle x-T^{n} x, p-T^{n} x\right\rangle \leq\left\|x-T^{n} x\right\|^{2}+\frac{\mu_{n}}{2} \phi(\|x-p\|)+\frac{\xi_{n}}{2} \\
\left\langle x-p, T^{n} x-p\right\rangle \leq\|x-p\|^{2}+\frac{\mu_{n}}{2} \phi(\|x-p\|)+\frac{\xi_{n}}{2}
\end{gathered}
$$

Lemma 5 (see [19]). Consider

(i) $\|x \pm y\|^{2}=\|x\|^{2} \pm 2\langle x, y\rangle+\|y\|^{2}$, for all $x, y \in H$;

(ii) $\|(1-t) x+t y\|^{2}=(1-t)\|x\|^{2}+t\|y\|^{2}-t(1-t)\|x-y\|^{2}$, for all $x, y \in H$ and $t \in \mathbb{R}$.

Lemma 6 (see [18]). Let $C$ be a bounded and closed convex subset of a real Hilbert space $H$. Let $T: C \rightarrow C$ be a uniformly L-Lipschitz and total quasi-asymptotically pseudocontractive mapping with $\operatorname{Fix}(T) \neq \emptyset$. Suppose there exist positive constants $M$ and $M^{*}$, for the function $\phi$ in (13), $\phi(\zeta) \leq M^{*} \zeta^{2}$ for all $\zeta \geq M$ such that

$$
\phi(\zeta) \leq \phi(M)+M^{*} \zeta^{2}
$$

Then $\operatorname{Fix}(T)$ is a closed convex subset of $C$.

Lemma 7 (see [20]). A mapping $I-T: C \rightarrow C$ is said to be demiclosed at zero, if for any sequence $\left\{x_{n}\right\} \in C$, such that $x_{n} \rightarrow x^{*} \in C$ and $(I-T) x_{n} \rightarrow 0$ as $n \rightarrow \infty$; then $(I-T) x^{*}=$ 0 .

Lemma 8 (see [21]). Let $\left\{r_{n}\right\},\left\{s_{n}\right\}$, and $\left\{t_{n}\right\}$ be sequences of nonnegative real numbers satisfying

$$
r_{n+1} \leq\left(1+t_{n}\right) r_{n}+s_{n}, \quad n \geq 1
$$

If $\sum_{n=1}^{\infty} t_{n}<\infty$ and $\sum_{n=1}^{\infty} s_{n}<\infty$, then the limit $\lim _{n \rightarrow \infty} r_{n}$ exists.

Lemma 9 (see [22]). Let a sequence $\left\{t_{n}\right\} \in[0,1)$ satisfy $\lim _{n \rightarrow \infty} t_{n}=0$ and $\sum_{n=1}^{\infty} t_{n}=\infty$. Let $\left\{a_{n}\right\}$ be a sequence of nonnegative real numbers that satisfies any of the following conditions.

(i) For all $\varepsilon>0$, there exists an integer $N \geq 1$ such that, for all $n \geq N$,

$$
a_{n+1} \leq\left(1-t_{n}\right) a_{n}+t_{n} \varepsilon
$$

(ii) $a_{n+1} \leq\left(1-t_{n}\right) a_{n}+o_{n}, n \geq 0$, where $o_{n} \geq 0$ satisfies $\lim _{n \rightarrow \infty} o_{n} / t_{n}=0$;

(iii) $a_{n+1} \leq\left(1-t_{n}\right) a_{n}+t_{n} c_{n}$, where $\overline{\lim }_{n \rightarrow \infty} c_{n} \leq 0$.

Then $\lim _{n \rightarrow \infty} a_{n}=0$.

\section{Main Results}

In this section, we will prove the strong convergence of (7) to solve the two-set SCFPP.

Theorem 10. Let $C$ and $Q$ be nonempty closed convex subsets of real Hilbert spaces $H_{1}$ and $H_{2}$, respectively. Let $U: H_{1} \rightarrow$ $H_{1}$ be a uniformly $k_{1}$-Lipschitz and $\left(\left\{\mu_{n}^{(1)}\right\},\left\{\xi_{n}^{(1)}\right\}, \phi_{1}\right)$-total quasi-asymptotically pseudocontractive mapping, $\mathrm{T}: \mathrm{H}_{2} \rightarrow$ $\mathrm{H}_{2}$ a uniformly $k_{2}$-Lipschitz, and $\left(\left\{\mu_{n}^{(2)}\right\},\left\{\xi_{n}^{(2)}\right\}, \phi_{2}\right)$-total quasiasymptotically pseudocontractive mappings satisfying the following conditions:

$$
\begin{aligned}
& \left(C_{1}\right) C:=\operatorname{Fix}(U) \neq \emptyset, Q:=\operatorname{Fix}(T)=\emptyset ; \\
& \left(C_{2}\right) \mu_{n}=\max \left\{\mu_{n}^{(1)}, \mu_{n}^{(2)}\right\}, \xi_{n}=\max \left\{\xi_{n}^{(1)}, \xi_{n}^{(2)}\right\}, n \geq 1, \text { and } \\
& \sum_{n=1}^{\infty} \mu_{n}<\infty, \sum_{n=1}^{\infty} \xi_{n}<\infty ; \\
& \left(C_{3}\right) \phi=\max \left\{\phi_{1}, \phi_{2}\right\} \text { and } \exists M, M^{*}>0 .
\end{aligned}
$$

Let $\psi: H_{1} \rightarrow H_{1}$ be a $\delta$-contraction with $\delta \in(0,1)$. Let $A: H_{1} \rightarrow H_{2}$ be a bounded linear operator. For $\forall x_{1} \in$ $H_{1}$, sequence $\left\{x_{n}\right\}$ can be generated by the iteration (7), where the sequence $\left\{\alpha_{n}\right\} \subset(0,1)$ satisfies (i) $\lim _{n \rightarrow \infty} \alpha_{n}=0$ and (ii) $\sum_{n=0}^{\infty} \alpha_{n}=\infty,\{\beta\} \subset[a, b]$ with $a, b \in\left(0,1 /\left(1+k_{1}\right)\right)$, and $\{\gamma\} \subset(0,2 / L)$ with $L$ being the largest eigenvalue of the matrix $A^{T} A$. Assume that $I-U$ and $I-T$ are demiclosed at zero. If $\Gamma \neq \emptyset$, then $\left\{x_{n}\right\}$ generated by (7) converges strongly to a solution of the two-set SCFPP.

Proof. (1) First of all, we show that, for $\forall p \in \Gamma,\left\{x_{n}\right\}$ generated by (7) is bounded.

From (7), (16), and Lemma 6, we have

$$
\begin{aligned}
\left\|u_{n}-p\right\|^{2}= & \left\|x_{n}-p+\gamma A^{*}\left(T^{n}-I\right) A x_{n}\right\|^{2} \\
= & \left\|x_{n}-p\right\|^{2}+\gamma^{2}\left\|A^{*}\left(I-T^{n}\right) A x_{n}\right\|^{2} \\
& +2 \gamma\left\langle A x_{n}-A p, T^{n} A x_{n}-A x_{n}\right\rangle \\
\leq & \left\|x_{n}-p\right\|^{2}+\gamma^{2} L^{2}\left\|\left(I-T^{n}\right) A x_{n}\right\|^{2} \\
& +\gamma \mu_{n} \phi\left(\left\|A x_{n}-A p\right\|\right)+\gamma \xi_{n} \\
\leq & \left(1+\gamma \mu_{n} M^{*} L\right)\left\|x_{n}-p\right\|^{2} \\
& +\gamma^{2} L^{2}\left\|\left(I-T^{n}\right) A x_{n}\right\|^{2}+\gamma \mu_{n} \phi(M)+\gamma \xi_{n} .
\end{aligned}
$$

Since

$$
\begin{aligned}
\left\|\left(I-T^{n}\right) A x_{n}\right\| & \leq\left\|A x_{n}-A p\right\|+\left\|T^{n} A x_{n}-A p\right\| \\
& \leq\left(\|A\|+k_{2}\|A\|\right)\left\|x_{n}-p\right\|,
\end{aligned}
$$

substituting (25) into (24), we have

$$
\begin{aligned}
\left\|u_{n}-p\right\|^{2} \leq & {\left[1+\gamma \mu_{n} M^{*} L+\gamma^{2} L^{2}\left(\|A\|+k_{2}\|A\|\right)^{2}\right] } \\
& \times\left\|x_{n}-p\right\|^{2}+\gamma \mu_{n} \phi(M)+\gamma \xi_{n} \\
= & \left(1+a_{n}\right)\left\|x_{n}-p\right\|^{2}+\gamma \mu_{n} \phi(M)+\gamma \xi_{n},
\end{aligned}
$$


where $a_{n}=\gamma \mu_{n} M^{*} L+\gamma^{2} L^{2}\left(\|A\|+k_{2}\|A\|\right)^{2}$; by condition $\left(C_{2}\right)$, we know

$$
\sum_{n=1}^{\infty} a_{n}<\infty .
$$

Next, from (7), (13), and Lemma 5, we can get

$$
\begin{aligned}
\left\|y_{n}-p\right\|^{2}= & \left\|(1-\beta)\left(u_{n}-p\right)+\beta\left(U^{n}\left(u_{n}\right)-p\right)\right\|^{2} \\
= & (1-\beta)\left\|u_{n}-p\right\|^{2}+\beta\left\|U^{n}\left(u_{n}\right)-p\right\|^{2} \\
& -\beta(1-\beta)\left\|u_{n}-U^{n}\left(u_{n}\right)\right\|^{2} \\
\leq & (1-\beta)\left\|u_{n}-p\right\|^{2}+\beta\left[\left\|u_{n}-p\right\|^{2}+\left\|u_{n}-U^{n}\left(u_{n}\right)\right\|^{2}\right. \\
& \left.+\mu_{n} \phi\left(\left\|u_{n}-p\right\|\right)+\xi_{n}{ }^{2}\right] \\
& -\beta(1-\beta)\left\|u_{n}-U^{n}\left(u_{n}\right)\right\|^{2} \\
= & \left\|u_{n}-p\right\|^{2}+\beta^{2}\left\|u_{n}-U^{n}\left(u_{n}\right)\right\|^{2} \\
& +\beta \mu_{n} \phi\left(\left\|u_{n}-p\right\|\right)+\beta \xi_{n} ;
\end{aligned}
$$

we also can see that

$$
\begin{aligned}
\left\|u_{n}-U^{n}\left(u_{n}\right)\right\| & \leq\left\|u_{n}-p\right\|+\left\|U^{n}\left(u_{n}\right)-p\right\| \\
& \leq\left(1+k_{1}\right)\left\|u_{n}-p\right\| ;
\end{aligned}
$$

then substituting (29) into (28) and from (26), we have

$$
\begin{aligned}
\left\|y_{n}-p\right\|^{2} \leq & {\left[1+\beta^{2}\left(1+k_{1}\right)^{2}\right]\left\|u_{n}-p\right\|^{2} } \\
& +\beta \mu_{n} \phi\left(\left\|u_{n}-p\right\|\right)+\beta \xi_{n} \\
\leq & {\left[1+\beta^{2}\left(1+k_{1}\right)^{2}+\beta \mu_{n} M^{*}\right]\left\|u_{n}-p\right\|^{2} } \\
& +\beta \mu_{n} \phi(M)+\beta \xi_{n} \\
\leq & \left(1+b_{n}\right)\left(1+a_{n}\right)\left\|x_{n}-p\right\|^{2}+\mu_{n} \phi(M) \\
& \times\left[\left(1+b_{n}\right) \gamma+\beta\right]+\xi_{n}\left[\left(1+b_{n}\right) \gamma+\beta\right],
\end{aligned}
$$

where $b_{n}=\beta^{2}\left(1+k_{1}\right)^{2}+\beta \mu_{n} M^{*}$, and we also know that

$$
\sum_{n=1}^{\infty} b_{n}<\infty .
$$

From (7) and Lemma 5, we also have

$$
\begin{aligned}
\left\|x_{n+1}-p\right\|^{2}= & \left\|\alpha_{n}\left[\psi\left(y_{n}\right)-p\right]+\left(1-\alpha_{n}\right)\left(y_{n}-p\right)\right\|^{2} \\
\leq & \alpha_{n}\left\|\psi\left(y_{n}\right)-p\right\|^{2}+\left(1-\alpha_{n}\right)\left\|y_{n}-p\right\|^{2} \\
\leq & \left(1+2 \alpha_{n} \delta\right)\left\|y_{n}-p\right\|^{2} \\
& +2 \alpha_{n}\|\psi(p)-p\|^{2}-\alpha_{n}\left\|y_{n}-p\right\|^{2} \\
\leq & \left(1+2 \alpha_{n} \delta\right)\left\|y_{n}-p\right\|^{2}+2 \alpha_{n}\|\psi(p)-p\|^{2} .
\end{aligned}
$$

Substituting (30) into (32) and simplifying it we have

$$
\begin{aligned}
\left\|x_{n+1}-p\right\|^{2} \leq & \left(1+2 \alpha_{n} \delta\right)\left(1+b_{n}\right)\left(1+a_{n}\right) \\
& \times\left\|x_{n}-p\right\|^{2}+2 \alpha_{n}\|\psi(p)-p\|^{2} \\
& +\left(1+2 \alpha_{n} \delta\right)\left\{\mu_{n} \phi(M)\left[\left(1+b_{n}\right) \gamma+\beta\right]\right. \\
& \left.+\xi_{n}\left[\left(1+b_{n}\right) \gamma+\beta\right]\right\} \\
= & {\left[1+a_{n}+\left(1+a_{n}\right)\left(b_{n}+2 a_{n} \delta+2 a_{n} b_{n} \delta\right)\right] } \\
& \times\left\|x_{n}-p\right\|^{2}+2 \alpha_{n}\|\psi(p)-p\|^{2} \\
+ & +\left(1+2 \alpha_{n} \delta\right)\left\{\mu_{n} \phi(M)\left[\left(1+b_{n}\right) \gamma+\beta\right]\right. \\
& \left.+\xi_{n}\left[\left(1+b_{n}\right) \gamma+\beta\right]\right\} ;
\end{aligned}
$$

set

$$
\begin{gathered}
t_{n}=a_{n}+\left(1+a_{n}\right)\left(b_{n}+2 a_{n} \delta+2 a_{n} b_{n} \delta\right), \\
s_{n}=2 \alpha_{n}\|\psi(p)-p\|^{2}+\left(1+2 \alpha_{n} \delta\right) \\
\times\left\{\mu_{n} \phi(M)\left[\left(1+b_{n}\right) \gamma+\beta\right]+\xi_{n}\left[\left(1+b_{n}\right) \gamma+\beta\right]\right\} ;
\end{gathered}
$$

(33) can be rewritten as

$$
\left\|x_{n+1}-p\right\|^{2} \leq\left(1+t_{n}\right)\left\|x_{n}-p\right\|^{2}+s_{n}
$$

by condition $\left(C_{2}\right),(27)$, and (31), we know that $\sum_{n=1}^{\infty} t_{n}<\infty$ and $\sum_{n=1}^{\infty} s_{n}<\infty$. Thus it follows from Lemma 8 that the following limit exists:

$$
\lim _{n \rightarrow \infty}\left\|x_{n}-p\right\|
$$

Therefore, we obtain that $\left\{x_{n}\right\}$ is bounded, so is $\left\{u_{n}\right\}$. Set $z_{n}=$ $U^{n}\left(u_{n}\right)$. Then $\left\{z_{n}\right\}$ is also bounded.

(2) Next we prove $\lim _{n \rightarrow \infty}\left\|x_{n+1}-x_{n}\right\|=0$, $\lim _{n \rightarrow \infty}\left\|u_{n+1}-u_{n}\right\|=0$.

For each $n \geq 1, \forall u_{n} \in H_{1}$, assume there exists $v_{i}^{(n)} \in$ $C(i=1,2)$ such that $u_{n}=w v_{1}^{(n)}+(1-w) v_{2}^{(n)}$ for $w \in(0,1)$. Then for all $q \in C$, and by virtue of (16), we have

$$
\begin{aligned}
\left\|u_{n}-U^{n}\left(u_{n}\right)\right\|^{2}= & \left\langle u_{n}-U^{n}\left(u_{n}\right), u_{n}-U^{n}\left(u_{n}\right)\right\rangle \\
= & \frac{1}{\beta}\left\langle u_{n}-y_{n}, u_{n}-U^{n}\left(u_{n}\right)\right\rangle \\
= & \frac{1}{\beta}\left\langle u_{n}-U^{n}\left(u_{n}\right)-\left(y_{n}-U^{n}\left(y_{n}\right)\right), u_{n}-y_{n}\right\rangle \\
& +\frac{1}{\beta}\left\langle y_{n}-U^{n}\left(y_{n}\right), u_{n}-y_{n}\right\rangle \\
\leq & \frac{1}{\beta}\left(\left\|u_{n}-y_{n}\right\|+\left\|U^{n}\left(u_{n}\right)-U^{n}\left(y_{n}\right)\right\|\right) \\
& \times\left\|u_{n}-y_{n}\right\|+\frac{1}{\beta}\left\langle u_{n}-q, y_{n}-U^{n}\left(y_{n}\right)\right\rangle
\end{aligned}
$$




$$
\begin{aligned}
& +\frac{1}{\beta}\left\langle q-y_{n}, y_{n}-U^{n}\left(y_{n}\right)\right\rangle \\
\leq & \frac{1+k_{1}}{\beta}\left\|u_{n}-y_{n}\right\|^{2}+\frac{1}{\beta}\left\langle u_{n}-q, y_{n}-U^{n}\left(y_{n}\right)\right\rangle \\
& +\frac{1}{\beta}\left[\frac{\mu_{n}}{2} \phi\left(\left\|y_{n}-q\right\|\right)+\frac{\xi_{n}}{2}\right] \\
\leq & \left(1+k_{1}\right) \beta\left\|u_{n}-U^{n}\left(u_{n}\right)\right\|^{2} \\
& +\frac{1}{\beta}\left\langle u_{n}-q, y_{n}-U^{n}\left(y_{n}\right)\right\rangle \\
& +\frac{1}{\beta}\left[\frac{\mu_{n}}{2}\left[M^{*}\left\|y_{n}-q\right\|^{2}+\phi(M)\right]+\frac{\xi_{n}}{2}\right],
\end{aligned}
$$

which implies that

$$
\begin{aligned}
\beta[1 & \left.-\left(1+k_{1}\right) \beta\right]\left\|u_{n}-U^{n}\left(u_{n}\right)\right\|^{2} \\
\leq & \left\langle u_{n}-z, y_{n}-U^{n}\left(y_{n}\right)\right\rangle \\
& +\left[\frac{\mu_{n}}{2}\left[M^{*}\left\|y_{n}-z\right\|^{2}+\phi(M)\right]+\frac{\xi_{n}}{2}\right] .
\end{aligned}
$$

Now we take $q=v_{i}^{(n)}(i=1,2)$ in (38); multiplying $w$ and $(1-w)$ on the two side of (38), respectively, and then adding up, we can obtain

$$
\begin{aligned}
\beta & {\left[1-\left(1+k_{1}\right) \beta\right]\left\|u_{n}-U^{n}\left(u_{n}\right)\right\|^{2} } \\
& \leq\left[\frac{\mu_{n}}{2}\left[M^{*}\left\|y_{n}-z\right\|^{2}+\phi(M)\right]+\frac{\xi_{n}}{2}\right] .
\end{aligned}
$$

Letting $n \rightarrow \infty$ in (39), we have

$$
\lim _{n \rightarrow \infty}\left\|u_{n}-U^{n}\left(u_{n}\right)\right\|=0 .
$$

From (7), we know that

$$
\begin{aligned}
\left\|x_{n+1}-p\right\|^{2}= & \left\|y_{n}-p+\alpha_{n}\left(\psi\left(y_{n}\right)-y_{n}\right)\right\|^{2} \\
= & \left\|y_{n}-p\right\|^{2}+2 \alpha_{n}\left\langle y_{n}-p, \psi\left(y_{n}\right)-y_{n}\right\rangle \\
& +\alpha_{n}^{2}\left\|\psi\left(y_{n}\right)-y_{n}\right\|^{2} .
\end{aligned}
$$

Letting $n \rightarrow \infty$ in (41) and by condition (i) in Theorem 10, we know

$$
\lim _{n \rightarrow \infty}\left\|x_{n}-p\right\|=\lim _{n \rightarrow \infty}\left\|y_{n}-p\right\|
$$

Similarly,

$$
\begin{aligned}
\left\|y_{n}-p\right\|^{2}= & \left\|u_{n}-p+\beta\left(u_{n}-U^{n}\left(u_{n}\right)\right)\right\|^{2} \\
= & \left\|u_{n}-p\right\|^{2}+2 \beta\left\langle u_{n}-p, u_{n}-U^{n}\left(u_{n}\right)\right\rangle \\
& +\beta^{2}\left\|u_{n}-U^{n}\left(u_{n}\right)\right\|^{2}
\end{aligned}
$$

from (40) the limit of $\left\|y_{n}-p\right\|$ exists and

$$
\lim _{n \rightarrow \infty}\left\|x_{n}-p\right\|=\lim _{n \rightarrow \infty}\left\|y_{n}-p\right\|=\lim _{n \rightarrow \infty}\left\|u_{n}-p\right\| .
$$

Therefore, when we take limit on both sides of (22), we can deduce that

$$
\lim _{n \rightarrow \infty}\left\|A x_{n}-T^{n} A x_{n}\right\|=0 .
$$

Then,

$$
\begin{aligned}
\left\|x_{n+1}-x_{n}\right\| \leq & \left\|y_{n}-x_{n}\right\|+\alpha_{n}\left\|\psi\left(y_{n}\right)-y_{n}\right\| \\
\leq & \left\|u_{n}-x_{n}\right\|+\beta\left\|u_{n}-U^{n}\left(u_{n}\right)\right\| \\
& +\alpha_{n}\left\|\psi\left(y_{n}\right)-y_{n}\right\| \\
\leq & \gamma\|A\|\left\|A x_{n}-T^{n} A x_{n}\right\|+\beta\left\|u_{n}-U^{n}\left(u_{n}\right)\right\| \\
& +\alpha_{n}\left\|\psi\left(y_{n}\right)-y_{n}\right\| .
\end{aligned}
$$

In view of (40) and (45) we have that

$$
\lim _{n \rightarrow \infty}\left\|x_{n+1}-x_{n}\right\|=0
$$

Similarly, it follows from (7), (45), and (47) that

$$
\begin{aligned}
& \left\|u_{n+1}-u_{n}\right\| \\
& =\left\|x_{n+1}-x_{n}-\gamma A^{*}\left(I-T^{n+1}\right) A x_{n+1}-\gamma A^{*}\left(I-T^{n}\right) A x_{n}\right\| \\
& \leq\left\|x_{n+1}-x_{n}\right\|+\gamma\left\|A^{*}\right\|\left\|A x_{n+1} T^{n+1}-A x_{n+1}\right\| \\
& \quad+r\left\|A^{*}\right\|\left\|A x_{n} T^{n}-A x_{n}\right\| \longrightarrow 0(n \longrightarrow \infty) .
\end{aligned}
$$

(3) Next we prove that $\left\|x_{n}-U\left(u_{n}\right)\right\| \rightarrow 0$, as $n \rightarrow \infty$. From (40) and (48), we have

$$
\begin{aligned}
& \left\|u_{n}-U\left(u_{n}\right)\right\| \\
& \leq\left\|u_{n}-U^{n}\left(u_{n}\right)\right\|+\left\|U^{n}\left(u_{n}\right)-U\left(u_{n}\right)\right\| \\
& \leq\left\|u_{n}-U^{n}\left(u_{n}\right)\right\|+k_{1}\left\|U^{n-1}\left(u_{n}\right)-u_{n}\right\| \\
& \leq\left\|u_{n}-U^{n}\left(u_{n}\right)\right\|+k_{1}\left[\left\|U^{n-1}\left(u_{n}\right)-U^{n-1}\left(u_{n-1}\right)\right\|\right. \\
& \left.\qquad+\left\|U^{n-1}\left(u_{n-1}\right)-u_{n}\right\|\right] \\
& \leq\left\|u_{n}-U^{n}\left(u_{n}\right)\right\|+k_{1}^{2}\left\|u_{n}-u_{n-1}\right\| \\
& +k_{1}\left[\left\|U^{n-1}\left(u_{n-1}\right)-u_{n-1}\right\|+\left\|u_{n-1}-u_{n}\right\|\right] \\
& \longrightarrow \infty(n \rightarrow \infty) .
\end{aligned}
$$
that

By the same way, from (45) and (47) we can also prove

$$
\left\|A x_{n}-T A x_{n}\right\| \longrightarrow 0, \quad n \longrightarrow \infty .
$$


Therefore, from (44) and (49), we know

$$
\begin{aligned}
\left\|x_{n}-U\left(u_{n}\right)\right\| & \leq\left\|x_{n}-u_{n}\right\|+\left\|u_{n}-U\left(u_{n}\right)\right\| \\
& \longrightarrow 0 \quad(n \longrightarrow \infty) .
\end{aligned}
$$

Since $\left\{x_{n}\right\}$ is bounded, there exists a subsequence $\left\{x_{n_{i}}\right\}$ of $\left\{x_{n}\right\}$ which converges weakly to a point $x^{*}$. Without loss of generality, we may assume that $\left\{x_{n}\right\}$ converges weakly to $x^{*}$. Therefore, from (49)-(51) and Lemma 7, we have $x^{*} \in$ $\operatorname{Fix}(U)$.

(4) Finally, we prove that $x_{n} \rightarrow x^{*}$ in norm. To do this, we calculate

$$
\begin{aligned}
& \left\|x_{n+1}-x^{*}\right\|^{2} \\
& =\left\langle\alpha_{n} \psi\left(y_{n}\right)+\left(1-\alpha_{n}\right) y_{n}-x^{*}, x_{n+1}-x^{*}\right\rangle \\
& =\left\langle\alpha_{n} \psi\left(y_{n}\right)+\left(1-\alpha_{n}\right) y_{n}-x^{*}, x_{n+1}-x^{*}\right\rangle \\
& =\alpha_{n}\left\langle\psi\left(y_{n}\right)-x^{*}, x_{n+1}-x^{*}\right\rangle \\
& +\left(1-\alpha_{n}\right)\left\langle y_{n}-x^{*}, x_{n+1}-x^{*}\right\rangle \\
& \leq \alpha_{n}\left\langle\psi\left(y_{n}\right)-\psi\left(x^{*}\right), x_{n+1}-x^{*}\right\rangle \\
& +\alpha_{n}\left\langle\psi\left(x^{*}\right)-x^{*}, x_{n+1}-x^{*}\right\rangle \\
& +\frac{1-\alpha_{n}}{2}\left\|y_{n}-x^{*}\right\|^{2}+\frac{1-\alpha_{n}}{2}\left\|x_{n+1}-x^{*}\right\|^{2} \\
& \leq \frac{\alpha_{n} \delta}{2}\left\|y_{n}-x^{*}\right\|^{2}+\frac{\alpha_{n}}{2}\left\|x_{n+1}-x^{*}\right\|^{2} \\
& +\alpha_{n}\left\langle\psi\left(x^{*}\right)-x^{*}, x_{n+1}-x^{*}\right\rangle \\
& +\frac{1-\alpha_{n}}{2}\left\|y_{n}-x^{*}\right\|^{2}+\frac{1-\alpha_{n}}{2}\left\|x_{n+1}-x^{*}\right\|^{2} \\
& =\frac{1-(1-\delta) \alpha_{n}}{2}\left\|y_{n}-x^{*}\right\|^{2}+\frac{1}{2}\left\|x_{n+1}-x^{*}\right\|^{2} \\
& +\alpha_{n}\left\langle\psi\left(x^{*}\right)-x^{*}, x_{n+1}-x^{*}\right\rangle \text {. }
\end{aligned}
$$

Therefore, we have

$$
\begin{aligned}
\left\|x_{n+1}-x^{*}\right\|^{2} \leq & \left(1-(1-\delta) \alpha_{n}\right)\left\|y_{n}-x^{*}\right\|^{2} \\
& +2 \alpha_{n}\left\langle\psi\left(x^{*}\right)-x^{*}, x_{n+1}-x^{*}\right\rangle .
\end{aligned}
$$

Substituting (23) into (28), we have

$$
\begin{aligned}
\left\|y_{n}-x^{*}\right\|^{2} \leq & \left\|x_{n}-p\right\|^{2}+\gamma^{2} L^{2}\left\|\left(I-T^{n}\right) A x_{n}\right\|^{2} \\
& +\beta^{2}\left\|u_{n}-U^{n}\left(u_{n}\right)\right\|^{2} \\
& +\mu_{n}\left[\gamma \phi\left(\left\|A x_{n}-A p\right\|\right)+\beta \phi\left(\left\|u_{n}-p\right\|\right)\right] \\
& +\xi_{n}(\gamma+\beta) .
\end{aligned}
$$

Since $(1-\delta) \alpha_{n} \in(0,1)$ and substituting (53) into (51), we get

$$
\begin{aligned}
\| x_{n+1} & -x^{*} \|^{2} \\
\leq & \left(1-(1-\delta) \alpha_{n}\right)\left\|x_{n}-p\right\|^{2} \\
& +\left(1-(1-\delta) \alpha_{n}\right) \\
\times & \left\{\gamma^{2} L^{2}\left\|\left(I-T^{n}\right) A x_{n}\right\|^{2}+\beta^{2}\left\|u_{n}-U^{n}\left(u_{n}\right)\right\|^{2}\right. \\
& \left.+\mu_{n}\left[\gamma \phi\left(\left\|A x_{n}-A p\right\|\right)+\beta \phi\left(\left\|u_{n}-p\right\|\right)\right]+\xi_{n}(\gamma+\beta)\right\} .
\end{aligned}
$$

Let

$$
\begin{aligned}
o_{n}= & \left(1-(1-\delta) \alpha_{n}\right) \\
\times & \left\{\gamma^{2} L^{2}\left\|\left(I-T^{n}\right) A x_{n}\right\|^{2}+\beta^{2}\left\|u_{n}-U^{n}\left(u_{n}\right)\right\|^{2}\right. \\
& \left.+\mu_{n}\left[\gamma \phi\left(\left\|A x_{n}-A p\right\|\right)+\beta \phi\left(\left\|u_{n}-p\right\|\right)\right]+\xi_{n}(\gamma+\beta)\right\} .
\end{aligned}
$$

Equation (55) can be rewritten as

$$
\left\|x_{n+1}-x^{*}\right\|^{2} \leq\left(1-(1-\delta) \alpha_{n}\right)\left\|x_{n}-p\right\|^{2}+o_{n} .
$$

Evidently, from (40), (45), and Lemma 9 (ii), we can conclude that $x_{n+1}-x^{*} \rightarrow 0(n \rightarrow \infty)$.

This completes the proof.

The following theorem can be concluded from Theorem 10 immediately.

Theorem 11. Let $C$ and $Q$ be nonempty closed convex subsets of real Hilbert spaces $H_{1}$ and $H_{2}$, respectively. Let $U: H_{1} \rightarrow$ $H_{1}$ be a uniformly $k_{1}$-Lipschitz and quasi-pseudocontractive mapping with $C:=\operatorname{Fix}(U) \neq \emptyset$. Let $T: H_{2} \rightarrow H_{2}$ be a uniformly $k_{2}$-Lipschitz and quasi-pseudocontractive mapping with $Q:=\operatorname{Fix}(T)=\emptyset$. Let $\psi: H_{1} \rightarrow H_{1}$ be a $\delta$-contraction with $\delta \in(0,1)$. Let $A: H_{1} \rightarrow H_{2}$ be a bounded linear operator. For $\forall x_{1} \in H_{1}$, sequence $\left\{x_{n}\right\}$ can be generated by the iteration:

$$
\begin{gathered}
u_{n}=x_{n}-\gamma A(I-T) A x_{n}, \\
y_{n}=(1-\beta) u_{n}+\beta U\left(u_{n}\right), \\
x_{n+1}=\alpha_{n} \psi\left(y_{n}\right)+\left(1-\alpha_{n}\right) y_{n}, \quad n \geq 1,
\end{gathered}
$$

where the sequence $\left\{\alpha_{n}\right\} \subset(0,1)$ satisfies $(i) \lim _{n \rightarrow \infty} \alpha_{n}=0$ and (ii) $\sum_{n=0}^{\infty} \alpha_{n}=\infty,\{\beta\} \subset[a, b]$ with $a, b \in\left(0,1 /\left(1+k_{1}\right)\right)$, and $\{\gamma\} \subset(0,2 / L)$ with $L$ being the largest eigenvalue of the matrix $A^{T} A$. Assume that $I-U$ and $I-T$ are demiclosed at zero. If $\Gamma \neq \emptyset$, then $\left\{x_{n}\right\}$ generated by (58) converges strongly to a solution of the two-set SCFPP.

Proof. For each $p \in \Gamma$, if we take $T=T^{n}, U=U^{n}, \mu_{n} \rightarrow 0$ and $\xi_{n} \rightarrow 0$, and follow the proof of Theorem 10, we can also prove that $\left\{x_{n}\right\}$ converges strongly to $x^{*} \in \Gamma$ by the same way. 
Remark 12. Algorithm (7) and Theorems 10 and 11 improve and extend the corresponding results of Censor and Segal [2], Moudafi [3, 4], Mohammed [5, 6], Chang et al. [11, 13], Yang et al. [12], and others.

\section{Concluding Remarks}

In this work, we develop the split common fixed point problem with more general classes of total quasi-asymptotically pseudocontractive and quasi-pseudocontractive operators; corresponding algorithms are improved based on the viscosity iteration; thus we can obtain strong convergence without more constraints on operators.

\section{Conflict of Interests}

The authors declare that there is no conflict of interests regarding the publication of this paper.

\section{Acknowledgments}

The authors would like to thank the associate editor and the referees for their comments and suggestions. This research was supported by the National Natural Science Foundation of China (11071053).

\section{References}

[1] Y. Censor and T. Elfving, "A multiprojection algorithm using Bregman projections in a product space," Numerical Algorithms, vol. 8, no. 2, pp. 221-239, 1994.

[2] Y. Censor and A. Segal, "The split common fixed point problem for directed operators," Journal of Convex Analysis, vol. 16, no. 2, pp. 587-600, 2009.

[3] A. Moudafi, "The split common fixed-point problem for demicontractive mappings," Inverse Problems, vol. 26, no. 5, Article ID 055007, 6 pages, 2010.

[4] A. Moudafi, "A note on the split common fixed-point problem for quasi-nonexpansive operators," Nonlinear Analysis: Theory, Methods \& Applications, vol. 74, no. 12, pp. 4083-4087, 2011.

[5] L. B. Mohammed, "A note on the split common fixed-point problem for strongly quasi-nonexpansive operator in Hilbert space," International Journal of Innovative Research and Studies, vol. 2, no. 8, pp. 424-434, 2013.

[6] L. B. Mohammed, "Strong convergence of an algorithm about quasi-nonexpansive mappings for the split common fixed-pint problem in Hilbert space," International Journal of Innovative Research and Studies, vol. 2, no. 8, pp. 298-306, 2013.

[7] D. Sheng and R. Chen, "On the strong convergence of an algorithm about pseudo-demicontractive mappings for the split common fixed-point problem," 2011, http://www.paper.edu.cn/.

[8] Y. Yu and D. Sheng, "On the strong convergence of an algorithm about firmly pseudo-demicontractive mappings for the split common fixed-point problem," Journal of Applied Mathematics, vol. 2012, Article ID 256930, 9 pages, 2012.

[9] G. Gu, S. Wang, and Y. J. Cho, "Strong convergence algorithms for hierarchical fixed points problems and variational inequalities," Journal of Applied Mathematics, vol. 2011, Article ID 164978, 17 pages, 2011.
[10] C. Zhang and S. He, "Strong convergence theorems for the split common fixed point problem for countable family of nonexpansive operators," Journal of Applied Mathematics, vol. 2012, Article ID 438121, 11 pages, 2012.

[11] S. S. Chang, L. Wang, Y. K. Tang, and L. Yang, "The split common fixed point problem for total asymptotically strictly pseudocontractive mappings," Journal of Applied Mathematics, vol. 2012, Article ID 385638, 13 pages, 2012.

[12] L. Yang, S. S. Chang, Y. J. Cho et al., "Multiple-set split feasibility problems for total asymptotically strict pseudocontraction mappings," Fixed Point Theory and Applications, vol. 2011, article 77, 2011.

[13] S.-S. Chang, Y. J. Cho, J. K. Kim, W. B. Zhang, and L. Yang, "Multiple-set split feasibility problems for asymptotically strict pseudocontractions," Abstract and Applied Analysis, vol. 2012, Article ID 491760, 12 pages, 2012.

[14] F. Wang and H.-K. Xu, "Cyclic algorithms for split feasibility problems in Hilbert spaces," Nonlinear Analysis: Theory, Methods \& Applications, vol. 74, no. 12, pp. 4105-4111, 2011.

[15] Y.-C. Tang, J.-G. Peng, and L.-W. Liu, "A cyclic algorithm for the split common fixed point problem of demicontractive mappings in Hilbert spaces," Mathematical Modelling and Analysis, vol. 17, no. 4, pp. 457-466, 2012.

[16] H. H. Bauschke and J. M. Borwein, "On projection algorithms for solving convex feasibility problems," SIAM Review, vol. 38, no. 3, pp. 367-426, 1996.

[17] L. Elsner, I. Koltracht, and M. Neumann, "Convergence of sequential and asynchronous nonlinear paracontractions," Numerische Mathematik, vol. 62, no. 1, pp. 305-319, 1992.

[18] Z.-M. Wang and Y. Su, "On the convergence of hybrid projection algorithms for total quasi-asymptotically pseudocontractive mapping," Journal of Inequalities and Applications, vol. 2013, article 375, 9 pages, 2013.

[19] G. Marino and H.-K. Xu, "Weak and strong convergence theorems for strict pseudo-contractions in Hilbert spaces," Journal of Mathematical Analysis and Applications, vol. 329, no. 1, pp. 336-346, 2007.

[20] K. Goebel and W. A. Kirk, Topics in Metric Fixed Point Theory, vol. 28 of Cambridge Studies in Advanced Mathematics, Cambridge University Press, Cambridge, UK, 1990.

[21] K. Aoyama, Y. Kimura, W. Takahashi, and M. Toyoda, "Approximation of common fixed points of a countable family of nonexpansive mappings in a Banach space," Nonlinear Analysis: Theory, Methods \& Applications, vol. 67, no. 8, pp. 2350-2360, 2007.

[22] J. G. O'Hara, P. Pillay, and H.-K. Xu, "Iterative approaches to finding nearest common fixed points of nonexpansive mappings in Hilbert spaces," Nonlinear Analysis: Theory, Methods \& Applications, vol. 54, no. 8, pp. 1417-1426, 2003. 


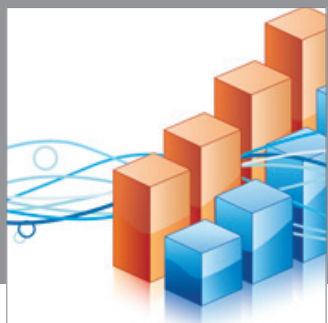

Advances in

Operations Research

mansans

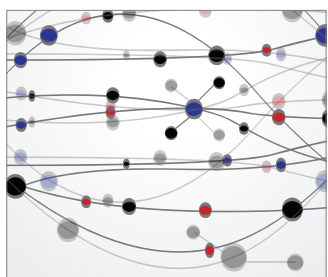

The Scientific World Journal
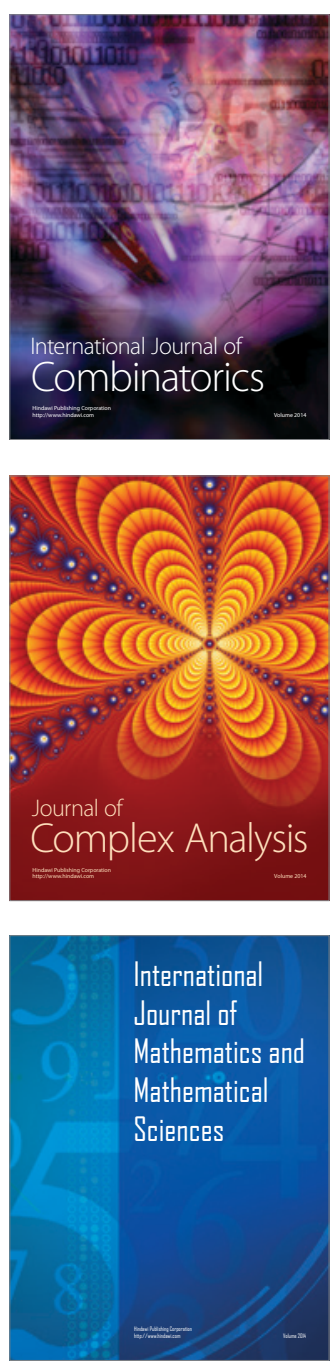
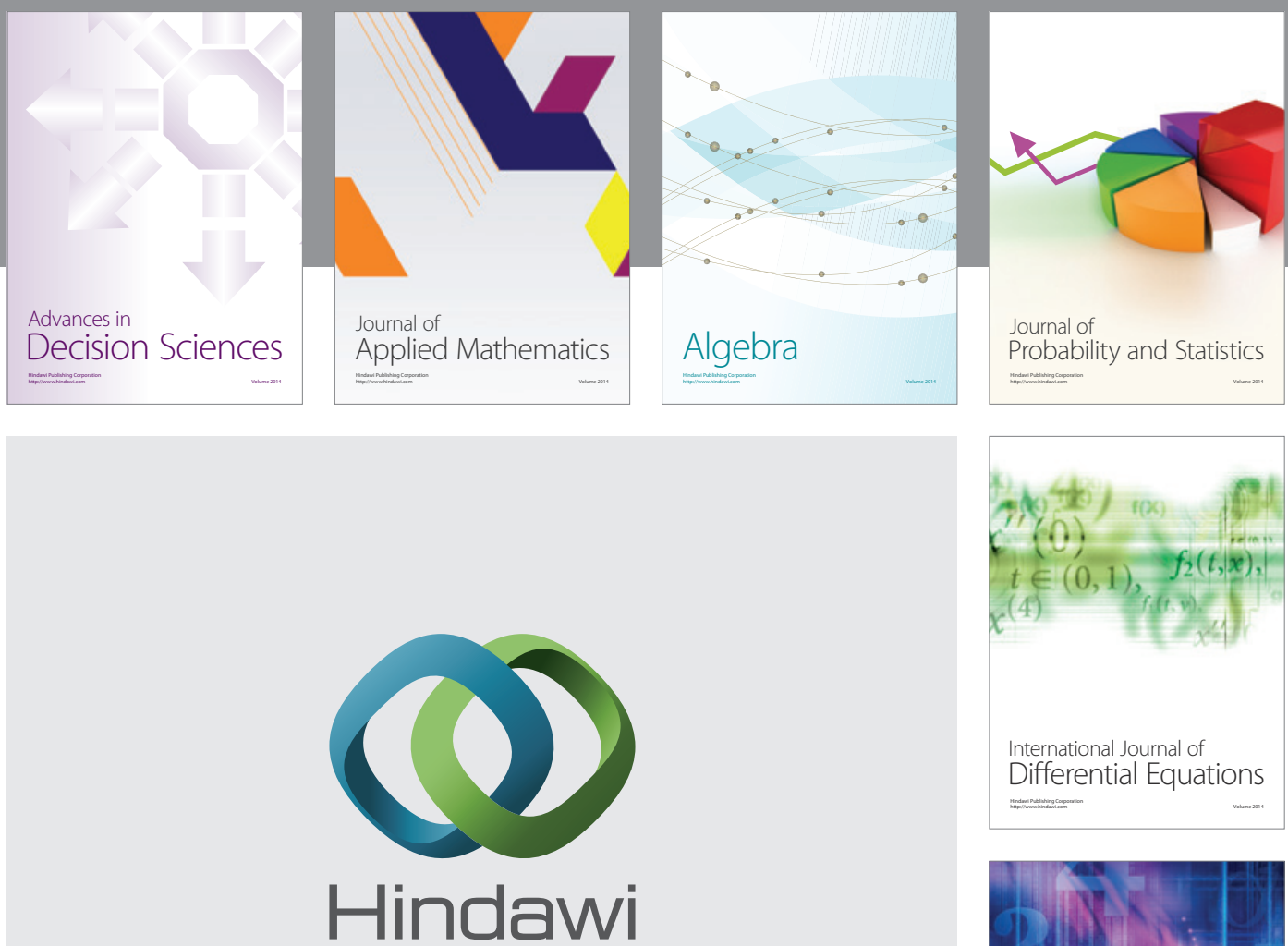

Submit your manuscripts at http://www.hindawi.com
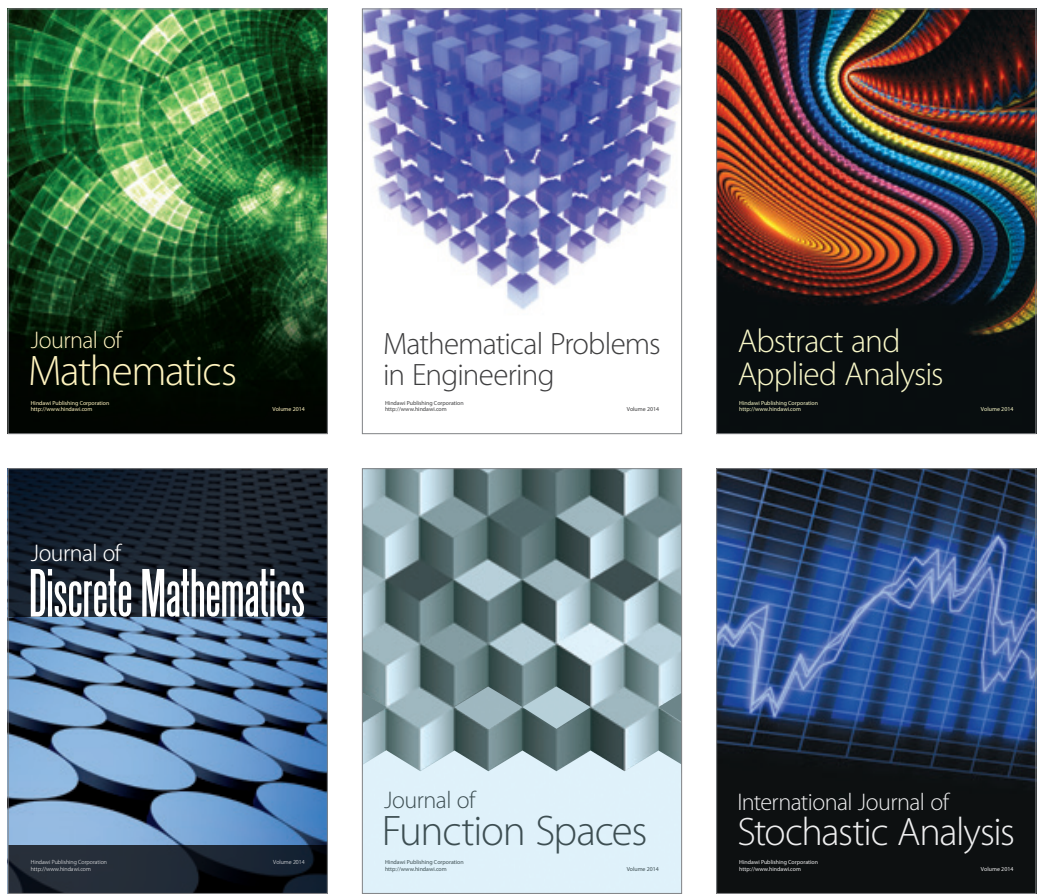

Journal of

Function Spaces

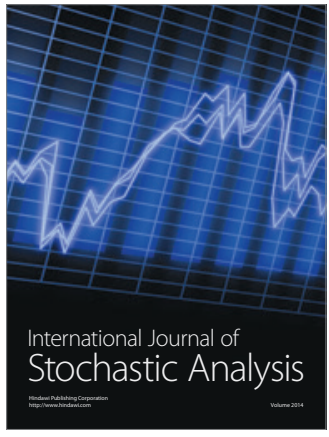

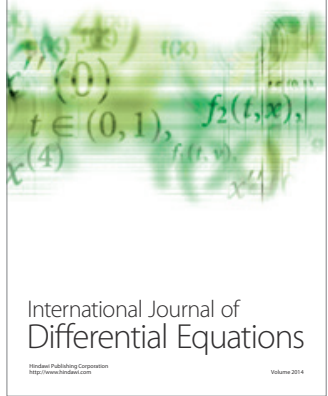
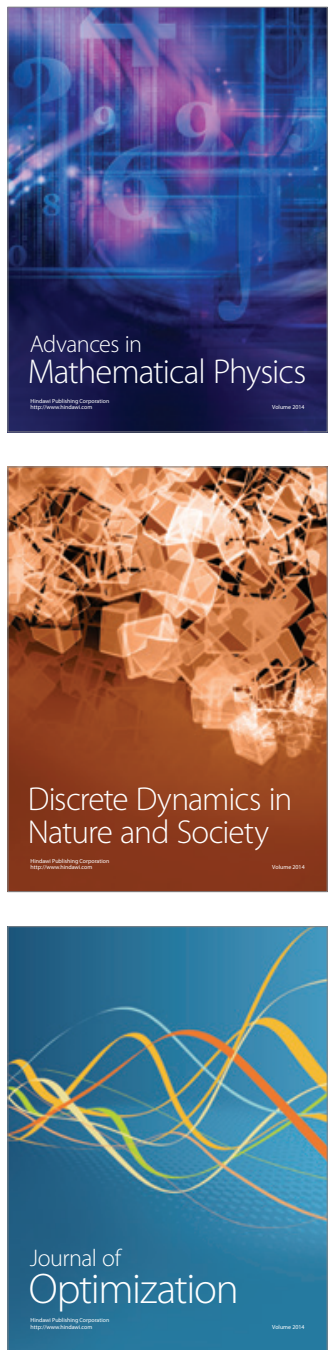